\title{
Lorlatinib: an additional option for ALK-positive non-small cell lung cancer?
}

\author{
Alice Mogenet, Pascale Tomasini, Laurent Greillier, Fabrice Barlesi \\ Department of Multidisciplinary Oncology \& Therapeutic Innovations, Aix Marseille University, CNRS, INSERM, CRCM, APHM, Marseille, \\ France \\ Correspondence to: Fabrice Barlesi, MD, PhD. Service d'Oncologie Multidisciplinaire et Innovations Thérapeutiques, Hôpital Nord, Chemin des \\ Bourrely, 13915 Marseille Cedex, France. Email: fabrice.barlesi@ap-hm.fr. \\ Provenance: This is an invited article commissioned by the Section Editor Hengrui Liang (Department of Thoracic Surgery, Guangzhou Medical \\ University, Guangzhou, China). \\ Comment on: Solomon BJ, Besse B, Bauer TM, et al. Lorlatinib in patients with ALK-positive non-small-cell lung cancer: results from a global phase \\ 2 study. Lancet Oncol 2018;19:1654-67.
}

Submitted Apr 16, 2019. Accepted for publication May 20, 2019.

doi: $10.21037 /$ tlcr.2019.05.10

View this article at: http://dx.doi.org/10.21037/tlcr.2019.05.10

\section{Introduction}

Metastatic non-small cell lung cancer (NSCLC) remains the first cause of cancer-related death worldwide (1). Recent progress in the medical treatment of NSCLC have been focusing on molecular alterations. Anaplastic lymphoma kinase $(A L K)$ rearrangements are found in about $3 \%$ to $5 \%$ of lung adenocarcinomas in western countries, more frequently non-smokers and younger patients than most NSCLC. $E M L 4-A L K$ represent the commonest fusion partner with around $30 \%$ gene fusions identified (2). The development of $A L K$ tyrosine kinase inhibitors (ALK-TKIs) over chemotherapy improved the response rate up to $60 \%$ in first line treatment and also the median survival for metastatic disease. Crizotinib represent the first generation of ALK-TKI and showed efficacy in second line treatment over Pemetrexed and Docetaxel (3) followed by first line assessment over platinum-based chemotherapy since 2014 (4). More recently, new generation ALK-TKIs, Ceritinib, Alectinib and Brigatinib showed efficacy in second then first line treatment $(5,6)$.

Unfortunately, all patients finally progress within one to two years challenging Crizotinib, due to various resistance mechanisms: $A L K$-dependent mutations, such as $L 1196 M$ or G1269A, ALK-amplifications, or most frequently ALKindependent mechanisms, mostly bypass mechanisms (7). These findings induced the development of new targeted therapies. Indeed, despite the good efficacy of postCrizotinib second generation ALK-TKIs $(8,9)$, patients inevitably progress, with an evolution of the resistance profiles. The leading mechanism of resistance at this time of the disease became the ALK-dependent G1202R mutation, for more than $20 \%$ of patients (10). Considering these findings, molecular monitoring with new histology or cytology samples at the time of progression are required to identify the mechanisms of resistance and guide further-line treatment (11).

Lung cancer is the first cause of central nervous system (CNS) metastases, which are deeply worsening the prognosis despite the recent development of many local and systemic treatments. Moreover, $A L K$ rearrangements showed a strong association with CNS metastases at the initial time of the disease $(35 \%)$ and CNS progression is common under first and further line treatment (60\%) (12). Consequently, CNS efficacy will be a major challenge to develop new ALK-TKIs and to adjust the therapeutic sequence.

Brigatinib and Lorlatinib represents the next-generation of ALK-TKIs, targeting several resistance mutations of first and second generation of treatment, particularly G1202R. Brigatinib showed good systemic and CNS efficacy in a phase II study conducted in a Crizotinib-resistant population, with an overall response rate (ORR) of 54\% (13). A phase III study of front-line setting Brigatinib compared to Crizotinib is currently going (NCT02737501). Lorlatinib 
Table 1 Systemic efficacy of Lorlatinib in the ALK-positive expansion cohorts of the phase II trial in Crizotinib naïve and pretreated population

\begin{tabular}{lcccc}
\hline Expansion cohorts & $\begin{array}{c}\text { Number of } \\
\text { patients }\end{array}$ & Population & ORR (95\% Cl), \% & Median PFS (95\% Cl), months \\
\hline EXP 1 & 30 & Treatment naive & $90(73.5-97.9)$ & NR (11.4-NR) \\
EXP 2-3A & 59 & Crizotinib resistant & $69.5(56.1-80.8)$ & NR (12.5-NR) \\
EXP 3B & 32 & Non-Crizotinib resistant & $32.1(15.9-52.4)$ & $5.5(2.7-9)$ \\
EXP 4-5 & 111 & Two or three prior ALK-TKIs resistant & $38.7(29.6-48.5)$ & $7.3(5.6-11)$ \\
\hline
\end{tabular}

ORR, overall response rate; PFS, progression free survival; EXP, expansion cohort; NR, not reached.

(PF-06463922; Pfizer Oncology, Groton, CT, USA) is a novel ALK-TKI developed specifically to target secondgeneration ALK-TKIs resistant mutants with optimal brain penetration. This paper will summarize and comment the global phase II trial leaded by Solomon et al. and published in 2018, and discuss the place of Lorlatinib among the fan of ALK-TKIs in a near future (14).

\section{Molecular characteristics}

Lorlatinib is a small ATP-competitive macrocyclic ALK-TKI. The macrocyclic formation showed invitro an improvement of the metabolic stability and a low propensity for p-glycoprotein 1 mediated efflux (15), leading respectively to an efficacy in ALK wild type and ALK dependent resistant NSCLC, and a better blood brain barrier penetration. Especially, preclinical studies highlighted impressive efficacy targeting the highly resistant G1202R mutant (16).

\section{Clinical activity}

Lorlatinib development has been quickly conducted in a phase I-II trial, leading to an accelerated approval from the US Food and Drug Administration (FDA) on Nov, 2, 2018 for ALK-rearranged patients who experienced disease progression under second-generation ALK-TKI.

First in human Lorlatinib administration was conducted over a phase I trial, multicenter single-arm open-label dose escalation trial assessing safety, maximum tolerated dose and antitumor efficacy (17). Dose escalation was conducted initially from 10 to $200 \mathrm{mg}$ once daily and 35 to $100 \mathrm{mg}$ twice daily. The $100 \mathrm{mg}$ once daily dose taken continuously in 21-day cycles was select for the phase II part of the trial.

The phase I part of the trial included 54 patients, including 41 ALK-positive NSCLC, all pretreated by ALK-TKIs or chemotherapy. The ORR was 46\% (19/41)
(95\% CI: 31-63\%) for overall population, 57\% (95\% CI: $29-82 \%)$ for NSCLC pretreated with one prior ALK-TKI and $42 \%$ (95\% CI: $23-63 \%$ ) for NSCLC pretreated with two or more ALK-TKIs. The estimated median progression free survival (PFS) was 9.6 months (95\% CI: 3.4-16.6): 13.5 and 9.2 months if patients were treated with one or more prior ALK-TKI, respectively.

Based on these findings, Lorlatinib was evaluated in the phase II part of the trial (14), a multicenter single-arm open-label trial assessing objective tumor response and intracranial tumor response in pooled subgroups of ALKpositive patients. Patients were divided in 6 expansion cohorts (EXP) depending on the ALK or ROS1 status and previous treatment history. Results were collected by scheduled clinical visits (Day 1, 8, 15 and every 3 months) and imaging assessment (CT scan of the chest, abdomen and pelvis and brain MRI every 6 weeks). A total of 275 patients were enrolled across all cohorts, outcomes about systemic efficacy are summarized in Table 1. In the pooled population of 198 patients receiving at least one ALK-TKI (EXP 2-5), the ORR was 47\% (95\% CI: $39.9-54.2)$ and median PFS was 7.3 months (95\% CI: 5.6-11).

\section{CNS efficacy}

Knowing the high frequency, morbidity and mortality due to CNS metastases in this population of ALK-rearranged NSCLC, CNS efficacy is considered as a major outcome for the next-generation ALK-TKIs development. Both part of the phase I-II trial included patients with asymptomatic treated or untreated CNS metastases. In the phase I study, CNS target lesions (measurable according to RECIST 1.1) were presents at baseline in 24 patients, 19 in the ALK-positive population. Among them, overall intracranial response (OIR) was $42 \%$ (95\% CI: 20-67\%). In the phase II study, CNS metastases were present at baseline in 8 of $30(27 \%)$ naïve-treatment patients (EXP1) and in 133 of 
198 (67\%) pretreated patients (EXP 2-5), most of whom locally pretreated. In EXP1 population, 3 patients had measurable baseline CNS lesions and OIR was 66.7\% (95\% CI: 9.4-99.2\%). In EXP 2-5 population, 81 patients had measurable baseline CNS lesions and OIR was 63\% (95\% CI: $51.5-73.4 \%)$. In the Crizotinib pretreated population (EXP 2-3A), OIR was $87 \%$ (95\% CI: 66.4-97.2\%). In the population pretreated by non-Crizotinib ALK-TKI (EXP 3B), OIR was 55.6\% (95\% CI: 21.2-86.3). In the population pretreated with at least two ALK-TKIs, OIR was $53.1 \%$ (95\% CI: 38.3-67.5\%). Despite the higher rate of intracranial response over systemic response, authors proved that overall response wasn't drive only by CNS efficacy.

\section{Safety and tolerability}

The multiplication of available treatments for ALK-positive NSCLC with prolonged response imply an extended exposure to ALK-TKIs in the targeted population, explaining the major importance of safety and tolerability outcomes. At the chosen dose of $100 \mathrm{mg}$ once daily, the most common adverse event was hypercholesterolemia, occurring in $81 \%$ of treated patients. Others adverse event were described: hypertriglyceridemia, peripheral neuropathy, peripheral edema, cognitive effects, weight gain, mood effect and asthenia. Most side effects were common to other targeted therapies but dyslipidemia $(81 \%$ of patients, divided in $66 \%$ grade $1-2,14 \%$ grade 3 and $1 \%$ grade 4$)$ and cognitive effects ( $18 \%$ of patients, divided in $17 \%$ grade $1-2$ and $1 \%$ grade 3 ) seemed to be unique to Lorlatinib. Even if dose interruption and dose reduction due to treatment-related adverse event occurred for $30 \%$ and $22 \%$ of patients respectively, permanent discontinuation was rare (3\%) and essentially related to cognitive effects.

\section{New treatments, new issues: choose the therapeutic schedule for ALK-positive NSCLC}

In this phase II trial, Lorlatinib showed impressive efficacy in ALK-positive NSCLC, whether pretreated or not, partially due to the large range of targeted molecular alterations. Unfortunately, molecular profile wasn't required at baseline and available data not showed. Nevertheless, preclinical data and retrospective results of the phase I study suggested an impressive efficacy targeting the highly resistant G1202R mutation, and most of the ALKdependent mutations implied in Crizotinib resistance
(L1196M, G1269A). Lorlatinib efficacy in more than one third of patients pretreated with a second generation ALKTKI (Alectinib, Ceritinib) with extended survival shows that next-generation TKIs will find their place in the spectrum of ALK inhibitors, leading to impressive median survival exceeding five years for ALK-positive NSCLC (18).

Liquid biopsy follow-up used in this trial could be an interesting further option in order to avoid repetitive invasive acts in this frail population, and will be particularly helpful knowing the high rate of CNS progression under ALK-TKIs (19). However, we regret the absence of results concerning iterative liquid biopsies and hope data will be discussed in an ancillary paper.

Inevitably, the matter of Lorlatinib resistance will be a major stake to help determine the best therapeutic schedule. First data on the subject highlighted double mutations (essentially C1156Y/L1198F) (20) or activation of the MET pathway (21), which might restore sensitivity to Crizotinib.

To conclude, Lorlatinib showed great systemic and CNS efficacy in ALK-positive NSCLC and a phase III trial is actually ongoing to compare Crizotinib with Lorlatinib in first line setting (CROWN trial, NCT03052608). The outcomes will certainly help to determine the future place of Lorlatinib in the complex therapeutic schedule for ALKpositive NSCLC. Assessment of a survival benefit in front line setting will probably lead to a quick FDA approval, but a direct comparison with other next generation ALK-TKIs (Alectinib, Ceritinib, Brigatinib) will be needed to precisely define the best therapeutic schedule. In case of negative issues in first line assessment, Lorlatinib will still be a relevant option during the disease course of ALKpositive NSCLC due to his impressive molecular spectrum. The results of the French IFCT-1803 LORLATU trial (NCT03727477) evaluating efficacy of treatment sequences in patients with NSCLC receiving Lorlatinib will also be helpful to determine his place among the fan of ALK-TKIs.

\section{Acknowledgments}

None.

\section{Footnote}

Conflicts of Interest: F Barlesi: Astra-Zeneca, Bayer, BristolMyers Squibb, Boehringer-Ingelheim, Eli Lilly Oncology, F. Hoffmann-La Roche Ltd, Novartis, Merck, MSD, Pierre Fabre, Pfizer and Takeda. The other authors have no conflicts of interest to declare. 


\section{References}

1. Siegel R, Ma J, Zou Z, et al. Cancer statistics, 2014. CA Cancer J Clin 2014;64:9-29.

2. Chia PL, Mitchell P, Dobrovic A, et al. Prevalence and natural history of ALK positive non-small-cell lung cancer and the clinical impact of targeted therapy with ALK inhibitors. Clin Epidemiol 2014;6:423-32.

3. Shaw AT, Kim DW, Nakagawa K, et al. Crizotinib versus chemotherapy in advanced ALK-positive lung cancer. N Engl J Med 2013;368:2385-94.

4. Solomon BJ, Mok T, Kim DW, et al. First-Line Crizotinib versus Chemotherapy in ALK -Positive Lung Cancer. N Engl J Med 2014;371:2167-77.

5. Soria JC, Tan DSW, Chiari R, et al. First-line ceritinib versus platinum-based chemotherapy in advanced ALKrearranged non-small-cell lung cancer (ASCEND-4): a randomised, open-label, phase 3 study. Lancet 2017;389:917-29.

6. Peters S, Camidge DR, Shaw AT, et al. Alectinib versus Crizotinib in Untreated ALK-Positive Non-Small-Cell Lung Cancer. N Engl J Med 2017;377:829-38.

7. Lin JJ, Riely GJ, Shaw AT. Targeting ALK: Precision Medicine Takes on Drug Resistance. Cancer Discov 2017;7:137-55.

8. Shaw AT, Gandhi L, Gadgeel S, et al. Alectinib in ALKpositive, crizotinib-resistant, non-small-cell lung cancer: a single-group, multicentre, phase 2 trial. Lancet Oncol 2016;17:234-42.

9. Shaw AT, Kim DW, Mehra R, et al. Ceritinib in ALKrearranged non-small-cell lung cancer. $\mathrm{N}$ Engl J Med 2014;370:1189-97.

10. Gainor JF, Dardaei L, Yoda S, et al. Molecular Mechanisms of Resistance to First- and Second-Generation ALK Inhibitors in ALK-Rearranged Lung Cancer. Cancer Discov 2016;6:1118-33.

11. Planchard D, Popat S, Kerr K, et al. Metastatic non-small cell lung cancer: ESMO Clinical Practice Guidelines for diagnosis, treatment and follow-up. Ann Oncol 2018;29:iv192-237.

Cite this article as: Mogenet A, Tomasini P, Greillier L, Barlesi F. Lorlatinib: an additional option for ALK-positive non-small cell lung cancer? Transl Lung Cancer Res 2019;8(Suppl 4):S383S386. doi: 10.21037/tlcr.2019.05.10
12. Guérin A, Sasane M, Zhang J, et al. Brain metastases in patients with ALK+ non-small cell lung cancer: clinical symptoms, treatment patterns and economic burden. J Med Econ 2015;18:312-22.

13. Kim DW, Tiseo M, Ahn MJ, et al. Brigatinib in Patients With Crizotinib-Refractory Anaplastic Lymphoma KinasePositive Non-Small-Cell Lung Cancer: A Randomized, Multicenter Phase II Trial. J Clin Oncol 2017;35:2490-8.

14. Solomon BJ, Besse B, Bauer TM, et al. Lorlatinib in patients with ALK-positive non-small-cell lung cancer: results from a global phase 2 study. Lancet Oncol 2018;19:1654-67.

15. Akamine T, Toyokawa G, Tagawa T, et al. Spotlight on lorlatinib and its potential in the treatment of NSCLC: the evidence to date. Onco Targets Ther 2018;11:5093-101.

16. Zou HY, Friboulet L, Kodack DP, et al. PF-06463922, an ALK/ROS1 Inhibitor, Overcomes Resistance to First and Second Generation ALK Inhibitors in Preclinical Models. Cancer Cell 2015;28:70-81.

17. Shaw AT, Felip E, Bauer TM, et al. Lorlatinib in nonsmall-cell lung cancer with ALK or ROS1 rearrangement: an international, multicentre, open-label, single-arm firstin-man phase 1 trial. Lancet Oncol 2017;18:1590-9.

18. Duruisseaux M, Besse B, Cadranel J, et al. Overall survival with crizotinib and next-generation ALK inhibitors in ALK-positive non-small-cell lung cancer (IFCT-1302 CLINALK): a French nationwide cohort retrospective study. Oncotarget 2017;8:21903-17.

19. Hofman P. ALK Status Assessment with Liquid Biopsies of Lung Cancer Patients. Cancers 2017. doi: 10.3390/ cancers9080106.

20. Yoda S, Lin JJ, Lawrence MS, et al. Sequential ALK Inhibitors Can Select for Lorlatinib-Resistant Compound ALK Mutations in ALK-Positive Lung Cancer. Cancer Discov 2018;8:714-29.

21. Gouji T, Takashi S, Mitsuhiro T, et al. Crizotinib can overcome acquired resistance to $\mathrm{CH} 5424802$ : is amplification of the MET gene a key factor? J Thorac Oncol 2014;9:e27-8. 